\title{
BRM270 targets cancer stem cells and augments chemo-sensitivity in cancer (Review)
}

\author{
NISANSALA CHANDIMALI ${ }^{1,2^{*}}$, HYEBIN KOH $^{2,3^{*}}$, JIHWAN KIM $^{4}$, JAIHYUNG LEE $^{5}$, \\ YANG HO PARK ${ }^{6}$, HU-NAN SUN ${ }^{7}$ and TAEHO KWON ${ }^{8}$
}

\begin{abstract}
${ }^{1}$ Immunotherapy Convergence Research Center, Korea Research Institute of Bioscience and Biotechnology (KRIBB), Daejeon 34141; ${ }^{2}$ Department of Functional Genomics, University of Science and Technology, Daejeon 34113;

${ }^{3}$ Futuristic Animal Resource and Research Center, Korea Research Institute of Bioscience and Biotechnology (KRIBB), Cheongju-Si, Chungbuk 28116; ${ }^{4}$ Korean Convergence Medicine Centre, 100 years Oriental Medical Clinic, Seoul 04783; ${ }^{5}$ Epigenetics Drug Discovery Centre, Haeam Convalescence Hospital, Gyeonggi 12458; ${ }^{6}$ Evidencebased Medicine Centre, Park Yang Ho BRM Institute, Seoul 07163, Republic of Korea; ${ }^{7}$ College of Life Science and Technology, Heilongjiang Bayi Agricultural University, Daqing, Heilongjiang 163319, P.R. China; ${ }^{8}$ Primate Resources Center, Korea Research Institute of Bioscience and Biotechnology (KRIBB), Jeonbuk 56216, Republic of Korea
\end{abstract}

Received May 12, 2020; Accepted July 21, 2020

DOI: 10.3892/ol.2020.11964

\begin{abstract}
Over the past decade, a number of studies have demonstrated the resistance of cancer cells to conventional drugs and have recognized this as a major challenge in cancer therapy. While attempting to understand the underlying mechanisms of chemoresistance, several studies have suggested that the presence of cancer stem cells (CSCs) in tumors is one of the major pathways contributing toward resistance. Chemoresistance leads to cancer treatment failure and worsens the prognosis of patients. Natural herbal compounds are gaining attention as an alternative treatment strategy for cancer. These compounds may be effective against chemoresistant cells either alone or synergistically alongside conventional drugs, sensitizing cancer cells and enhancing the therapeutic efficacy. BRM270 is a natural compound made from seven herbal plant (Saururus chinensis, Citrus unshiu Markovich, Aloe vera, Arnebia euchroma, Portulaca oleracea, Prunella vulgaris var. lilacina and
\end{abstract}

Correspondence to: Dr Taeho Kwon, Primate Resources Center, Korea Research Institute of Bioscience and Biotechnology (KRIBB), 351-33 Neongme-gil, Ibam-myeon, Jeongeup-si, Jeonbuk 56216, Republic of Korea

E-mail: kwon@kribb.re.kr

Professor Hu-Nan Sun, College of Life Science and Technology, Heilongjiang Bayi Agricultural University, 2 Xingyanglu, Daqing, Heilongjiang 163319, P.R. China

E-mail: sunhunan76@163.com

*Contributed equally

Key words: cancer stem cell, chemoresistance, natural compound, BRM270, non-small cell lung cancer, multi-drug resistant
Scutellaria bacicalensis) extracts used in Asian traditional medicine and has the potential to target CSCs. Several studies have demonstrated the positive effects of BRM270 against chemoresistant cancer and its synergy alongside existing cancer drugs, including paclitaxel and gefitinib. These effects have been observed against various cancer types, including resistant non-small cell lung cancer (NSCLC), glioblastoma, multi-drug resistant osteosarcoma, cervical cancer, pancreatic cancer and hepatocarcinoma. The present review discusses the effects of BRM270 treatment against CSC-associated chemoresistance in common types of cancer.

\section{Contents}

1. Introduction

2. BRM270 overcomes chemoresistance by targeting cancer stem cells

3. Conclusions

\section{Introduction}

Emergence of primary and acquired resistance to existing conventional chemotherapies is one of the major challenges to overcome in the clinical management of cancer $(1,2)$. Patients with chemoresistant cancer do not respond to conventional drugs, which results in a poor prognosis and is often associated with the reoccurrence of malignancies following treatment $(3,4)$. Therefore, understanding the underlying molecular mechanisms of chemoresistance is necessary to improve the survival of these patients. The cancer stem cell (CSC) phenotype is one of the favored molecular mechanisms of chemoresistance (1). However, eradication of CSC populations to increase the therapeutic response of cancer cells to existing chemotherapies remains a challenge (5). Therefore, novel strategies to eliminate 
CSC populations and overcome chemoresistance are key for the successful clinical treatment of certain cancer types.

Herbal remedies have been used in traditional Asian medicine for centuries to treat various diseases. Recently, they have attracted global attention as potential strategies for overcoming the chemoresistance of cancer cells, without any observable side effects $(1,6)$. Medicinal plant extracts have the potential to treat human cancer either alone or synergistically with existing chemotherapeutic drugs to inhibit resistance (7-11). BRM270 (BRMLife) is a formulated extraction from seven medicinal plants used in Asian medicine, including Saururus chinensis, Citrus unshiu Markovich, Aloe vera, Arnebia euchroma, Portulaca oleracea, Prunella vulgaris var. lilacina and Scutellaria bacicalensis (Fig. 1) (1). Recently, the effectiveness of BRM270 was demonstrated as an alternative treatment to chemotherapy on non-small cell lung cancer (NSCLC) stem cells, which were resistant to EGFR-TKIs (epidermal growth receptor-specific tyrosine kinase inhibitors) (1). Similarly, previous studies have reported that BRM270 has the potential to downregulate tumorigenesis via the following mechanisms: Suppression of NF- $\mathrm{NB}$ signaling in multidrug resistance (MDR)-induced stem-like cells, inhibition of cervical CSC proliferation via SOX2 restriction, suppression of the recurrence and stem cell properties of glioblastoma, suppression of pancreatic CSC proliferation, and prevention of inflammation-promoted hepatocarcinogenesis (12-14).

Previous studies have also demonstrated the individual effects of the seven plant extract constituents of BRM270 (15-18). The present review discusses our current understanding of the individual and synergistic effects of BRM270 and highlights the important role of this compound in disrupting the CSC phenotype and overcoming the chemoresistance of cancer.

\section{BRM270 overcomes chemoresistance by targeting cancer stem cells}

BRM270 targets EGFR-TKI-resistant NSCLC stem cells. NSCLC includes adenocarcinomas, squamous cell carcinomas and large-cell carcinomas, accounting for $\sim 85 \%$ of all lung cancer cases $(19,20)$. The main treatment strategies for NSCLC are chemotherapy and radiotherapy, which fail to improve the survival rates of patients owing to primary and acquired resistance; therefore, only $15 \%$ of patients with NSCLC have a 5-year survival rate $(21,22)$. Inhibition of the EGFR tyrosine kinase pathway decreases NSCLC tumorigenesis, and EGFR-TKIs, including gefitinib and paclitaxel, are first-line treatments (1). However, the majority of patients with initial responses to EGFR-TKI treatment become resistant after 10-16 months (1). Resistance to the first generation of EGFR-TKIs has led to the development of novel drug generations and combination therapies $(1,22,23)$.

Due to the high resistance of NSCLCs to EGFR-TKIs, studies aiming to understand the mechanisms of resistance and to identify potential anti-resistance approaches remain necessary. Our recent study suggested a safe and effective alternative treatment involving the use of BRM270 for patients with EGFR-TKI-resistant NSCLC (1). The therapeutic efficacy of BRM270 against both non-resistant and EGFR-TKI-resistant lung adenocarcinoma cells was demonstrated (1). Resistance was derived from repeated exposure of lung adenocarcinoma cells to gefitinib and paclitaxel (1). It was demonstrated that BRM270 induced the apoptosis of chemoresistant NSCLC cells and caused $\mathrm{G}_{2} / \mathrm{M}$ cell cycle arrest via the inhibition of $\mathrm{NF}-\kappa \mathrm{B} / \mathrm{Bcl} 2$ signaling, thereby suppressing cell proliferation. Furthermore, it was demonstrated that BRM270 was capable of suppressing chemoresistant NSCLC stem cells by inhibiting epithelial-mesenchymal transition (EMT), metastasis and stemness (1). BRM270 was also reported to induce the expression of microRNA-128 (miR-128) in chemoresistant NSCLC (1). MicroRNA (miR)-128 is considered a tumor suppressor in various types of cancer (24-26), and acts against NSCLC cells by directly regulating vascular endothelial growth factor C (VEGF-C) (27). MiR-128 also induces lung cancer cell apoptosis by directly targeting NIMA (never in mitosis gene A)-related kinase 2 (28). Our previous study reported the tumor suppressive role of miR-128 in paclitaxel-resistant NSCLC by targeting MUC1-C and BMI-1 in CSCs (19). Furthermore, our previous study has suggested that miR-128 targets the c-met/PI3K/AKT pathway in lung CSCs and reverses gefitinib resistance (29). Therefore, BRM270 serves an important role in the prevention of the CSC phenotype, the inhibition of cancer progression and tumor growth, and the suppression of malignant behaviors in chemoresistant NSCLC through the induction of miR-128 overexpression (Fig. 2A) (1). Therefore, BRM270 is an effective compound that may be used to overcome chemoresistance in lung cancer as an alternative or addition to existing conventional drugs.

BRM270 suppresses the proliferation of cervical CSCs by inhibiting SOX2. Cervical cancer (CC) is the fourth most common cause of cancer-associated mortality in females worldwide (30). Surgical resection alone or in combination with adjuvant radiotherapy is commonly used to treat advanced stages of CC. However, this approach only decreases the risk of $\mathrm{CC}$ progression and is associated with irreversible morbidity (31). Due to the aggressive nature of CC, multimodality treatments are commonly used, including concurrent chemoradiotherapy or neoadjuvant chemotherapy (NACT) followed by surgery $(32,33)$. However, the application of these approaches, especially NACT, is restricted by the chemoresistance of CC cells to cisplatin, paclitaxel and taxel-based chemotherapies (31). Therefore, increasing the chemotherapeutic efficiency by targeting the underlying mechanisms of chemoresistance is essential for the successful clinical management of CC.

Previous studies have suggested that $\mathrm{CC}$ stem cells serve a crucial role in the resistance to conventional therapies (34-36). Our recent study reported that BRM270 inhibited CC stem cells by targeting SRY (sex determining region Y)-box 2 (SOX2) (37). SOX2 is a transcription factor that serves a crucial role in the maintenance of CSC stemness and the development of resistance to existing conventional therapies (38-41). As BRM270 has the potential to inhibit SOX2 expression, it can block the maintenance of the CSC phenotype from interfering with the emerging chemoresistance (37). BRM270 also negatively affects the progression and metastasis of $\mathrm{CC}$, the EMT and sphere formation of CC stem cells, and tumor initiation (37). It also promotes the apoptosis of CC cells by inhibiting cell proliferation. Furthermore, in CC stem cells, 


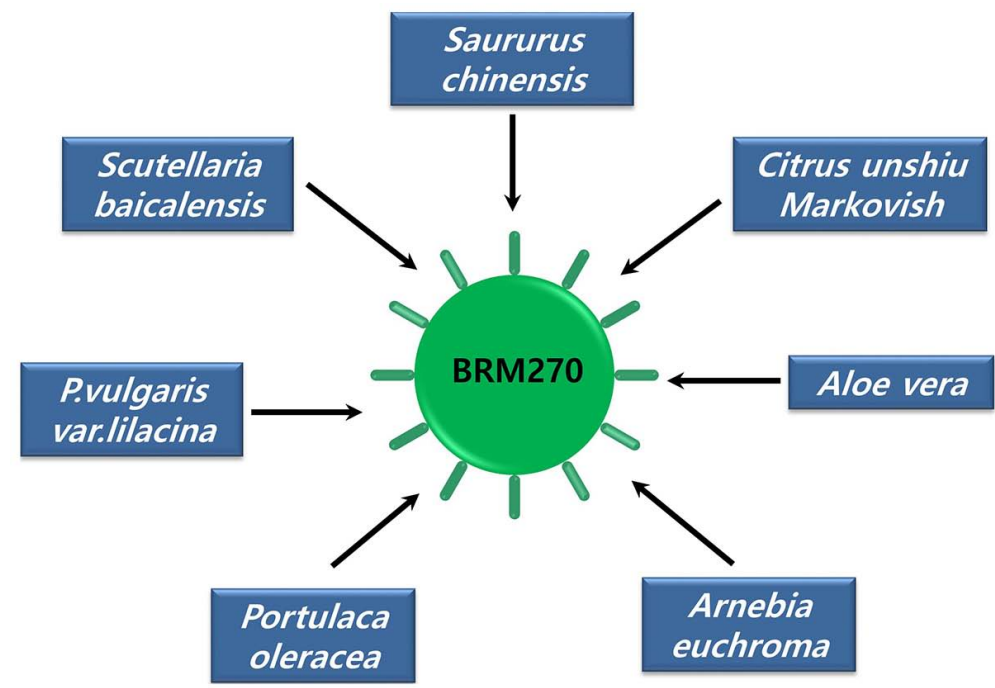

Figure 1. BRM270 consists of seven medicinal plant extracts. Saururus chinensis, Citrus unshiu Markovish, Aloe vera, Arnebia euchroma, Portulaca oleracea, Prunella vulgaris var. lilacina and Scutellaria baicalensis are the seven medicinal plants used in the BRM270 formulation.

BRM270 downregulates the Wnt/ß-Catenin pathway, which is essential to sustain the CSC phenotype (Fig. 2B) (37,42).

Therefore, BRM270 represents an effective alternative to the existing conventional therapies for cervical cancer by targeting CSCs to overcome chemoresistance, thereby improving clinical management.

BRM270 disrupts stem cell properties and synergizes with CCRT to prevent glioblastoma recurrence. Glioblastoma multiforme (GBM) is the most common and aggressive type of primary brain malignancy in adults (43). Vigorous multimodality treatments, including surgeries, concurrent temozolomide chemo-irradiation and post-radiotherapy adjuvant temozolomide are currently used to treat patients with glioblastoma (44). Despite these treatments, patient prognosis is poor and the 5-year survival rate is less than $10 \%$ following the initial diagnosis. Furthermore, the median survival time is 14.6 months owing to the infiltrative behavior and extreme resistance of cells to radiation and chemotherapy (45). This resistance also leads to the recurrence of glioblastoma following standard cancer therapy. Accumulating evidence suggests that glioblastoma stem cells are the major cause of chemo- and radio-resistance and are largely responsible for tumor recurrence (46-49). Therefore, the glioblastoma stem cell population is an important target for the successful clinical management of progressive and recurrent malignant glioblastoma.

A previous study demonstrated that BRM270 targets glioblastoma stem cells, suppressing growth and viability through the induction of apoptosis without affecting normal astrocytes (13). The sub- $\mathrm{G}_{0}$ cell population was significantly increased following the BRM270 treatment, indicating an expanded population of dead cells. Notably, BRM270 treatment did not cause any difference in $\mathrm{G}_{1}, \mathrm{~S}$ or $\mathrm{G}_{2} / \mathrm{M}$ subpopulations. Furthermore, the stemness properties of glioblastoma stem cells were also suppressed by treatment with BRM270. While BRM270 administration alone targeted the properties of glioblastoma stem cells, combined treatment with concurrent chemoradiotherapy (CCRT) successfully decreased the number of CD15-expressing stem cells and decreased the chance of glioblastoma recurrence (Fig. 2C).

Therefore, BRM270 alone and as a combined treatment with CCRT is effective in suppressing the CSC phenotype, overcoming resistance to radiotherapy and chemotherapy, and preventing the recurrence of malignant glioblastoma.

Pancreatic CSC proliferation is inhibited by BRM270 via the downregulation of sonic hedgehog signaling. Pancreatic ductal adenocarcinoma (PDAC) is considered one of the deadliest types of carcinoma, with a median survival time of less than 5-8 months (11). Gemcitabine is used as the first-line drug for PDAC, though intrinsic and acquired resistances remain major challenges. CSCs have been identified in previous studies as crucial players in the resistance of PDAC to existing conventional chemotherapy $(11,50)$.

Our recent study established that BRM270 was capable of inhibiting malignancy and the self-renewal capacity of CD44 ${ }^{+}$ PDAC cells (14). CD44 is a marker of the CSC population and the initiation of cancer, suggesting that BRM270 is able to inhibit CSC populations and stem-like properties of PDAC (51). BRM270 treatment also suppresses PDAC CSC-derived tumor growth via the downregulation of sonic hedgehog signaling in PDAC stem cells (14). The aberrant activation of the sonic hedgehog pathway is one of the major fundamental drivers of PDAC stem cell self-renewal and is associated with decreased survival rates in patients (Fig. 2D) (52-54).

Therefore, BRM270 is an effective treatment for PDAC and has the potential to reduce its resistance to existing chemotherapies by inhibiting PDAC stem cells, a crucial player in chemoresistance.

BRM270 inhibits tumorigenesis by suppressing $N F-\kappa B$ signaling in MDR-induced osteosarcoma stem cells. Osteosarcoma is an aggressive malignant tumor that primarily affects the skeletal system, and leads to a 5-year survival rate below $20 \%$ (55). It remains a challenge for current therapeutic strategies to effectively treat osteosarcoma due to the MDR of cancer cells to existing chemotherapeutic drugs, including doxorubicin and 


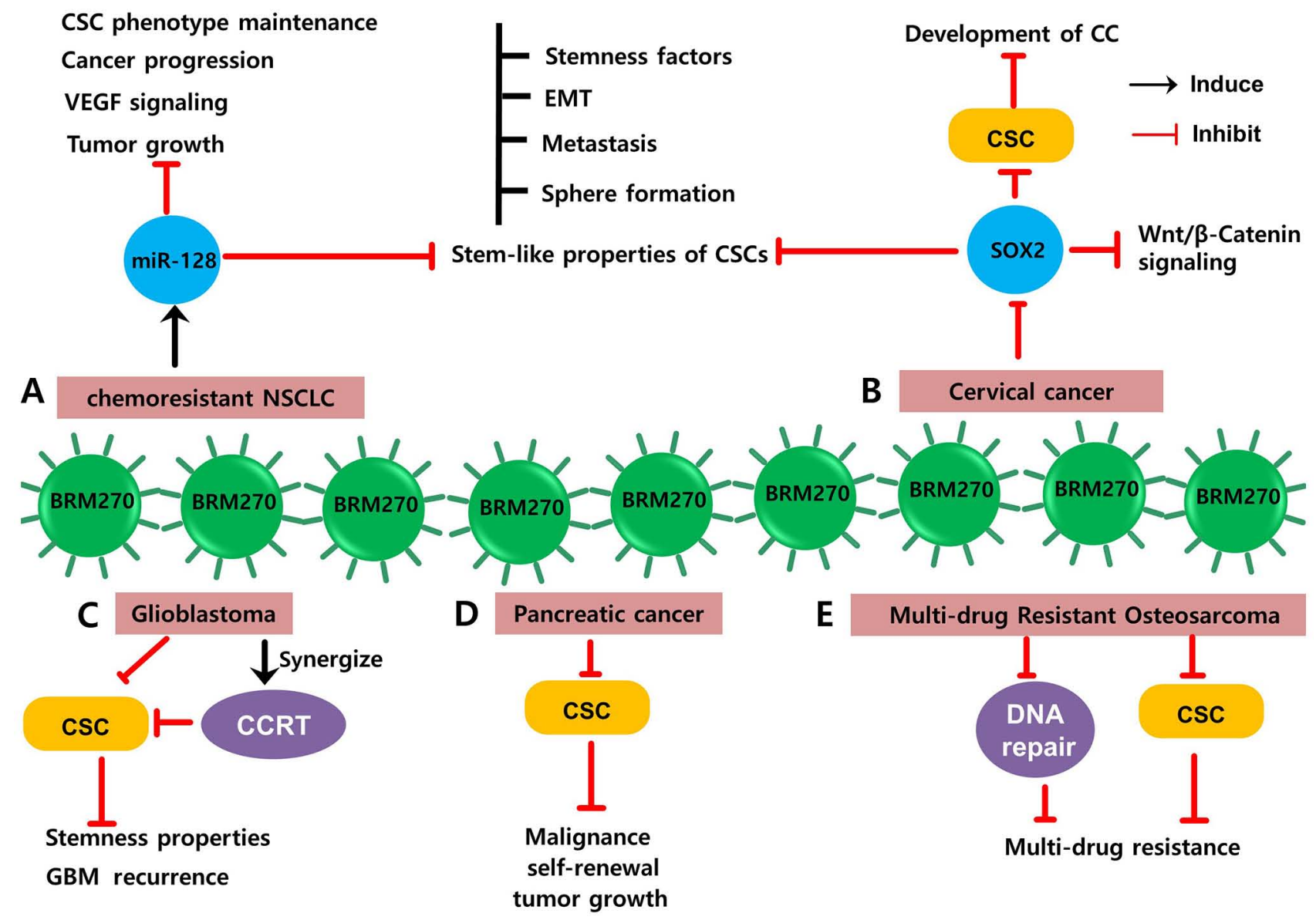

Figure 2. BRM270 acts against chemoresistance by inhibiting CSCs. BRM270 impacts gefitinib- and paclitaxel-resistant (A) NSCLC, (B) cervical cancer, (C) glioblastoma, (D) pancreatic cancer, and (E) multi-drug resistant osteosarcoma to overcome chemoresistance through the inhibition of CSC populations and stem-like characteristics. The CSC phenotype is one of the major underlying mechanisms in the chemoresistance of cancer cells. CSC, cancer stem cell; CC, cervical cancer; NSCLC, non-small cell lung cancer; VEGF, vascular endothelial growth factor; EMT, epithelial-mesenchymal transition; GBM, glioblastoma multiforme; CCRT, concurrent chemoradiotherapy; miR, microRNA; SOX2, SRY (sex determining region Y)-box 2.

cisplatin $(56,57)$. The presence of stem cell populations and altered DNA repair mechanisms have been identified as the major underlying methods of MDR in osteosarcoma $(57,58)$. Therefore, targeting these mechanisms would likely inhibit MDR and sensitize osteosarcoma cells to existing drugs.

The capability of BRM270 to circumvent MDR with minimal adverse side effects has been reported (12). BRM270 impacts osteosarcoma MDR by damaging DNA repair mechanisms and negatively affecting CSC populations (12). Our previous study demonstrated that BRM270 disrupts the formation of the microtubule cytoskeleton in doxorubicin-resistant osteosarcoma stem cells, inducing chromosomal condensation and nuclear fragmentation (12). BRM270 also induces programmed cell death and mitotic catastrophe in osteosarcoma stem cells by promoting irreversible DNA damage in the premature apoptosis stage and inhibiting cell proliferation selectively, without affecting the functions of normal cells (12). BRM270 treatment also induces the expression of key pro-apoptotic proteins, including structural maintenance of chromosome 2 (SMC2), Caspase-8, Interleukin-6 (IL-6), Cyclin-dependent kinase 6 (CDK6) and p65 (Fig. 2E) (12).

Therefore, BRM270 is a safe and effective natural compound to treat MDR osteosarcoma by promoting unrepairable DNA damage and cytotoxicity in osteosarcoma stem cells.
BRM270 prevents inflammation-inducedhepatocarcinogenesis. Hepatitis B virus (HBV), hepatitis C virus (HCV), non-alcoholic fatty liver disease, alcoholism or aflatoxin exposure are common causes of chronic hepatic injury (59). Persistent inflammation, as a result of chronic liver injury, is strongly associated with hepatocarcinogenesis, leading to the development of hepatocellular carcinoma (HCC) after years of inflammation (59). For example, the inability to clear HCV leads to chronic hepatitis C infection, resulting in inflammation-induced lesions in the liver, hepatic fat accumulation, and progressive fibrosis followed by HCC or cirrhosis (60-63). Chronic liver inflammation includes phorbal-12-myristate-13-acetate (PMA)-induced inflammation, which promotes hepatocarcinogenesis and HCC progression. This inflammation induces the expression of numerous inflammatory cytokines, including tumor necrosis factor alpha (TNF $\alpha$ ), Interleukin 1 (IL-1), IL-23, IL-6, cyclooxygenase-2 (COX-2), and lymphotoxins (LT) $\alpha$ and $\beta(59,64)$.

Our recent study reported that this enhanced BRM270 prevents PMA-induced inflammation through suppression of signal transducer and activator of transcription 3 (STAT3) signaling (14). As PMA is a COX-2 inducer and a mediator of inflammation, inhibition of PMA-induced inflammation by BRM270 decreases the expression of IL-6 and COX-2, leading to the suppression of hepatocarcinogenesis progression (14). 


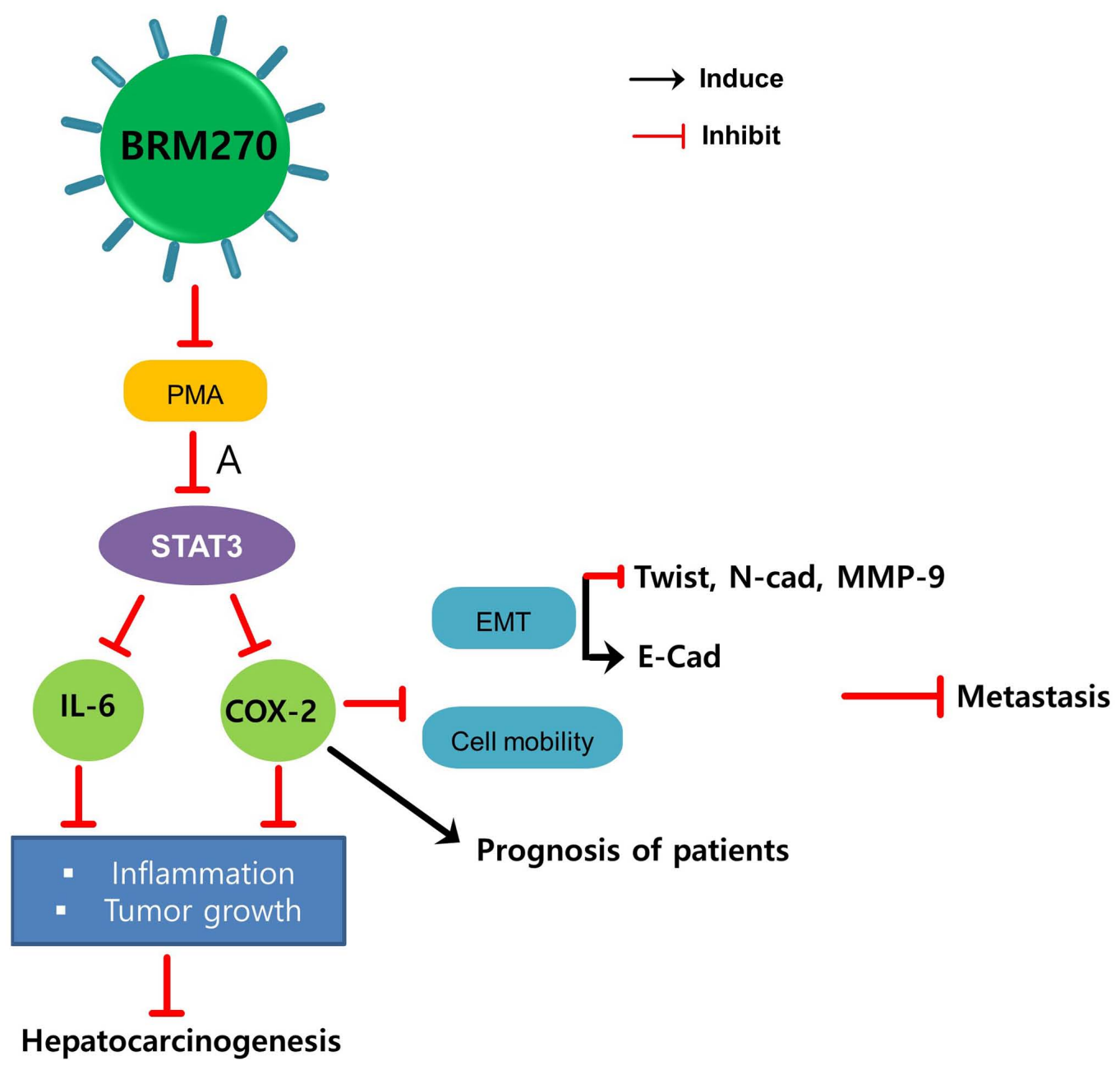

Figure 3. BRM270 suppresses inflammation-induced hepatocarcinogenesis by targeting the IL-6/STAT3/COX-2 axis. BRM270-induced PMA inhibits the phosphorylation of STAT3 at the tyrosine 705 site, thereby inhibiting IL-6 and COX-2 expression. IL- 6 and COX-2 are essential for tumor growth and inflammation-induced hepatocarcinogenesis. BRM270 also inhibits cell metastasis via the suppression of COX-2 and the reduction of EMT and cell mobility. PMA, phorbal-12-myristate-13-acetate; EMT, epithelial-mesenchymal transition; IL-6, interleukin 6; E/N-Cad, E/N-cadherin; COX-2, cyclooxygenase-2; MMP-9, matrix metallopeptidase 9.

BRM270 also inhibits hepatic tumor growth and HCC cell metastasis and improves the prognosis of patients with $\mathrm{HCC}$ via the inhibition of IL-6 and COX-2 expression (Fig. 3) (14).

Therefore, BRM270 is a natural compound treatment that has the potential to prevent hepatocarcinogenesis induced by inflammation through the downregulation of the IL-6/STAT3/COX-2 axis.

\section{Conclusions}

BRM270, a compound made of seven herbal plant extracts, is capable of negatively regulating the resistance of cancer cells to existing conventional therapies by inhibiting the CSC phenotype. BRM270 exerts its antitumor effects against chemoresistance via the suppression of CSC populations and the stem-like properties of CSCs. The compound can be used either as a standalone treatment or synergistically with existing drugs. Furthermore, the functionally enhanced product of BRM270 prevents inflammation-induced hepatocarcinogenesis. BRM270 does not cause any adverse side effects and does not affect normal human cells, but selectively targets cancer cells. Future studies should focus on further understanding the effects of BRM270 on CSCs and chemoresistance and investigate the possibility of BRM270 in clinical use.

\section{Acknowledgements}

Not applicable.

\section{Funding}

The present study was supported by the Basic Science Research Program through the National Research Foundation of Korea funded by the Ministry of Education, Korea (grant no. 2020R1I1 A2052417), the Korean Research Institute of Bioscience and Biotechnology Research Initiative Program, Korea (grant nos. KGM5162021 and RBM0112011), and the Scientific Research Team Support Plan of Heilongjiang Bayi Agricultural University, China (grant no. TDJH201904).

\section{Availability of data and materials}

Not applicable. 


\section{Authors' contributions}

NC, HK and TK contributed toward the conception of the study, writing the manuscript and performing the literature search. JK, JL and YHP conducted analysis and revised the manuscript. HNS and TK performed analysis and the quality assessment of the study. All authors read and approved the final manuscript.

\section{Ethics approval and consent to participate}

Not applicable.

\section{Patient consent for publication}

Not applicable.

\section{Competing interests}

The authors declare that they have no competing interests.

\section{References}

1. Kwon T, Chandimali N, Huynh DL, Zhang JJ, Kim N, Bak Y, Yoon DY, Yu DY, Lee JC, Gera M, et al: BRM270 inhibits cancer stem cell maintenance via microRNA regulation in chemoresistant A549 lung adenocarcinoma cells. Cell Death Dis 9: 244, 2018.

2. Toge M, Yokoyama S, Kato S, Sakurai H, Senda K, Doki Y, Hayakawa Y, Yoshimura N and Saiki I: Critical contribution of MCL-1 in EMT-associated chemo-resistance in A549 non-small cell lung cancer. Int J Oncol 46: 1844-1848, 2015.

3. Liu X, Han X, Wan X, He C, Wang Y, Mao A, Yu F, Zhou T, Feng L, Zhang P, et al: SPZ1 is critical for chemoresistance and aggressiveness in drug-resistant breast cancer cells. Biochem Pharmacol 156: 43-51, 2018.

4. Hudson AL, Weir C, Moon E, Harvie R, Klebe S, Clarke SJ, Pavlakis N and Howell VM: Establishing a panel of chemoresistant mesothelioma models for investigating chemo-resistance and identifying new treatments for mesothelioma. Sci Rep 4: 6152, 2014.

5. Roy L and Cowden Dahl KD: Can stemness and chemoresistance be therapeutically targeted via signaling pathways in ovarian cancer? Cancers (Basel) 10: 241, 2018.

6. Kim TW, Lee SY, Kim M, Cheon C, Jang BH, Shin YC and Ko SG: DSGOST regulates resistance via activation of autophagy in gastric cancer. Cell Death Dis 9: 649, 2018.

7. Chen Y, Zhu J and Zhang W: Antitumor effect of traditional Chinese herbal medicines against lung cancer. Anticancer Drugs 25: 983-991, 2014.

8. Ko JK and Auyeung KK: Target-oriented mechanisms of novel herbal therapeutics in the chemotherapy of gastrointestinal cancer and inflammation. Curr Pharm Des 19: 48-66, 2013.

9. Wang Q, Acharya N, Liu Z, Zhou X, Cromie M, Zhu J and Gao W: Enhanced anticancer effects of Scutellaria barbata D. Don in combination with traditional Chinese medicine components on non-small cell lung cancer cells. J Ethnopharmacol 217: 140-151, 2018.

10. Wang H, Wang F, Wu S, Liu Z, Li T, Mao L, Zhang J, Li C, Liu C and Yang Y: Traditional herbal medicine-derived sulforaphene promotes mitophagic cell death in lymphoma cells through CRM1-mediated p62/SQSTM1 accumulation and AMPK activation. Chem Biol Interact 281: 11-23, 2018.

11. Chandimali N, Huynh DL, Jin WY and Kwon T: Combination effects of hispidin and gemcitabine via inhibition of stemness in pancreatic cancer stem cells. Anticancer Res 38: 3967-3975, 2018.

12. Mongre RK, Sodhi SS, Ghosh M, Kim JH, Kim N, Park YH, Kim SJ, Heo YJ, Sharma N and Jeong DK: The novel inhibitor BRM270 downregulates tumorigenesis by suppression of NF-KB signaling cascade in MDR-induced stem like cancer-initiating cells. Int J Oncol 46: 2573-2585, 2015.
13. Jeon HY, Park CG, Ham SW, Choi SH, Lee SY, Kim JY, Seo S, Jin X, Kim JK, Eun K, et al: BRM270, a compound from natural plant extracts, inhibits glioblastoma stem cell properties and glioblastoma recurrence. J Med Food 20: 838-845, 2017.

14. Huynh DL, Koh H, Chandimali N, Zhang JJ, Kim N, Kang TY, Ghosh M, Gera M, Park YH, Kwon T and Jeong DK: BRM270 inhibits the proliferation of CD44 positive pancreatic ductal adenocarcinoma cells via downregulation of sonic hedgehog signaling. Evid Based Complement Alternat Med 2019: 8620469, 2019.

15. Choi BY, Joo JC, Lee YK, Jang IS, Park SJ and Park YJ: Anti-cancer effect of Scutellaria baicalensis in combination with cisplatin in human ovarian cancer cell. BMC Complement Altern Med 17: 277, 2017.

16. Lu C, Wang H, Chen S, Yang R, Li H and Zhang G: Baicalein inhibits cell growth and increases cisplatin sensitivity of A549 and H460 cells via miR-424-3p and targeting PTEN/PI3K/Akt pathway. J Cell Mol Med 22: 2478-2487, 2018.

17. Wang Y, Wang H, Zhou R, Zhong W, Lu S, Ma Z and Chai Y: Baicalin inhibits human osteosarcoma cells invasion, metastasis, and anoikis resistance by suppressing the transforming growth factor- $\beta 1$-induced epithelial-to-mesenchymal transition. Anticancer Drugs 28: 581-587, 2017.

18. Zhao R, Gao X, Cai Y, Shao X, Jia G, Huang Y, Qin X, Wang J and Zheng X: Antitumor activity of Portulaca oleracea L. polysaccharides against cervical carcinoma in vitro and in vivo. Carbohydr Polym 96: 376-383, 2013.

19. Koh H, Park H, Chandimali N, Huynh DL, Zhang JJ, Ghosh M, Gera M,Kim N,Bak Y, Yoon DY, et al: MicroRNA-128 suppresses paclitaxel-resistant lung cancer by inhibiting MUC1-C and BMI-1 in cancer stem cells. Oncotarget 8: 110540-110551, 2017.

20. Pazhouhandeh M, Samiee F, Boniadi T, Khedmat AF, Vahedi E, Mirdamadi M, Sigari N, Siadat SD, Vaziri F, Fateh A, et al: Comparative network analysis of patients with non-small cell lung cancer and smokers for representing potential therapeutic targets. Sci Rep 7: 13812, 2017.

21. Lam WK and Watkins DN: Lung cancer: Future directions. Respirology 12: 471-477, 2007.

22. Kwon T, Rho JK, Lee JC, Park YH, Shin HJ, Cho S, Kang YK, Kim BY, Yoon DY and Yu DY: An important role for peroxiredoxin II in survival of A549 lung cancer cells resistant to gefitinib. Exp Mol Med 47: e165, 2015.

23. Ham SY, Kwon T, Bak Y, Yu JH, Hong J, Lee SK, Yu DY and Yoon DY: Mucin 1-mediated chemo-resistance in lung cancer cells. Oncogenesis 5: e185, 2016.

24. Lin Y and Wu Z: MicroRNA-128 inhibits proliferation and invasion of glioma cells by targeting COX-2. Gene 658: 63-69, 2018.

25. Liang X, Shangguan W, Zhang M, Mei S, Wang L and Yang R: MiR-128 enhances dendritic cell-mediated anti-tumor immunity via targeting of p38. Mol Med Rep 16: 1307-1313, 2017.

26. Liu X, Liang Z, Gao K, Li H, Zhao G, Wang S and Fang J: MicroRNA-128 inhibits EMT of human osteosarcoma cells by directly targeting integrin $\alpha 2$. Tumour Biol 37: 7951-7957, 2016.

27. Hu J, Cheng Y, Li Y, Jin Z, Pan Y, Liu G, Fu S, Zhang Y, Feng K and Feng Y: MicroRNA-128 plays a critical role in human non-small cell lung cancer tumourigenesis, angiogenesis and lymphangiogenesis by directly targeting vascular endothelial growth factor-C. Eur J Cancer 50: 2336-2350, 2014.

28. Zhao D, Han W, Liu X, Cui D and Chen Y: MicroRNA-128 promotes apoptosis in lung cancer by directly targeting NIMA-related kinase 2. Thorac Cancer 8: 304-311, 2017.

29. Jiang J, Feng X, Zhou W, Wu Y and Yang Y: MiR-128 reverses the gefitinib resistance of the lung cancer stem cells by inhibiting the c-met/PI3K/AKT pathway. Oncotarget 7: 73188-73199, 2016.

30. Sikander M, Hafeez BB, Malik S, Alsayari A, Halaweish FT, Yallapu MM, Chauhan SC and Jaggi M: Cucurbitacin D exhibits potent anti-cancer activity in cervical cancer. Sci Rep 6: 36594, 2016.

31. Zhang J, Zhang Y, Liu S, Zhang Q, Wang Y, Tong L, Chen X, Ji Y, Shang Q, Xu B, et al: Metadherin confers chemoresistance of cervical cancer cells by inducing autophagy and activating ERK/NF- $\kappa B$ pathway. Tumour Biol 34: 2433-2440, 2013.

32. Burzawa J, Gonzales N and Frumovitz M: Challenges in the diagnosis and management of cervical neuroendocrine carcinoma. Expert Rev Anticancer Ther 15: 805-810, 2015.

33. Colombo $\mathrm{N}$ and Peiretti M: Critical review of neoadjuvant chemotherapy followed by surgery for locally advanced cervical cancer. Int J Gynecol Cancer 20 (11 Suppl 2): S47-S48, 2010.

34. Chhabra R: Cervical cancer stem cells: Opportunities and challenges. J Cancer Res Clin Oncol 141: 1889-1897, 2015. 
35. Huang R and Rofstad EK: Cancer stem cells (CSCs), cervical CSCs and targeted therapies. Oncotarget 8: 35351-35367, 2017.

36. Wang L, Guo H, Lin C, Yang L and Wang X: Enrichment and characterization of cancer stemlike cells from a cervical cancer cell line. Mol Med Rep 9: 2117-2123, 2014.

37. Chandimali N, Sun HN, Park YH and Kwon T: BRM270 suppresses cervical cancer stem cell characteristics and progression by inhibiting SOX2. In Vivo 34: 1085-1094, 2020.

38. Balahmar RM, Boocock DJ, Coveney C, Ray S, Vadakekolathu J, Regad T, Ali S and Sivasubramaniam S: Identification and characterisation of NANOG+/ OCT- $4^{\text {high }} / \mathrm{SOX} 2+$ doxorubicin-resistant stem-like cells from transformed trophoblastic cell lines. Oncotarget 9: 7054-7065, 2018.

39. Piva M, Domenici G, Iriondo O, Rábano M, Simões BM, Comaills V, Barredo I, López-Ruiz JA, Zabalza I, Kypta R and Vivanco Md: Sox2 promotes tamoxifen resistance in breast cancer cells. EMBO Mol Med 6: 66-79, 2014

40. Li Y, Chen K, Li L, Li R, Zhang J and Ren W: Overexpression of SOX2 is involved in paclitaxel resistance of ovarian cancer via the PI3K/Akt pathway. Tumour Biol 36: 9823-9828, 2015.

41. Jin Y, Jiang Z, Guan X, Chen Y, Tang Q, Wang G and Wang X: miR-450b-5p suppresses stemness and the development of chemoresistance by targeting SOX2 in colorectal cancer. DNA Cell Biol 35: 249-256, 2016.

42. Manzo-Merino J, Contreras-Paredes A, Vazquez-Ulloa E, Rocha-Zavaleta L, Fuentes-Gonzalez AM and Lizano M: The role of signaling pathways in cervical cancer and molecular therapeutic targets. Arch Med Res 45: 525-539, 2014.

43. Safa AR, Saadatzadeh MR, Cohen-Gadol AA, Pollok KE and Bijangi-Vishehsaraei K: Emerging targets for glioblastoma stem cell therapy. J Biomed Res 30: 19-31, 2016.

44. Zhu JJ and Wong ET: Personalized medicine for glioblastoma: Current challenges and future opportunities. Curr Mol Med 13: 358-367, 2013

45. Stupp R, Hegi ME, Mason WP, van den Bent MJ, Taphoorn MJ, Janzer RC, Ludwin SK, Allgeier A, Fisher B, Belanger K, et al: Effects of radiotherapy with concomitant and adjuvant temozolomide versus radiotherapy alone on survival in glioblastoma in a randomised phase III study: 5-year analysis of the EORTC-NCIC trial. Lancet Oncol 10: 459-466, 2009.

46. Garrido W, Rocha JD, Jaramillo C, Fernandez K, Oyarzun C, San Martin R and Quezada C: Chemoresistance in high-grade gliomas: Relevance of adenosine signalling in stem-like cells of glioblastoma multiforme. Curr Drug Targets 15: 931-942, 2014.

47. Bischof J, Westhoff MA, Wagner JE, Halatsch ME, Trentmann S, Knippschild U, Wirtz CR and Burster T: Cancer stem cells: The potential role of autophagy, proteolysis, and cathepsins in glioblastoma stem cells. Tumour Biol 39: 1010428317692227, 2017.

48. Uribe D, Torres A, Rocha JD, Niechi I, Oyarzún C, Sobrevia L, San Martín R and Quezada C: Multidrug resistance in glioblastoma stem-like cells: Role of the hypoxic microenvironment and adenosine signaling. Mol Aspects Med 55: 140-151, 2017.

49. Safari M and Khoshnevisan A: Cancer stem cells and chemoresistance in glioblastoma multiform: A review article. J Stem Cells 10: 271-285, 2015

50. Cioffi M, Trabulo SM, Sanchez-Ripoll Y, Miranda-Lorenzo I, Lonardo E, Dorado J, Reis Vieira C, Ramirez JC, Hidalgo M, Aicher A, et al: The miR-17-92 cluster counteracts quiescence and chemoresistance in a distinct subpopulation of pancreatic cancer stem cells. Gut 64: 1936-1948, 2015.
51. Ju SY, Chiou SH and Su Y: Maintenance of the stemness in CD44(+) HCT-15 and HCT-116 human colon cancer cells requires miR-203 suppression. Stem Cell Res 12: 86-100, 2014.

52. Rosow DE, Liss AS, Strobel O, Fritz S, Bausch D, Valsangkar NP, Alsina J, Kulemann B, Park JK, Yamaguchi J, et al: Sonic hedgehog in pancreatic cancer: From bench to bedside, then back to the bench. Surgery 152 (3 Suppl 1): S19-S32, 2012.

53. Song L, Chen X, Wang P, Gao S, Qu C and Liu L: Effects of baicalein on pancreatic cancer stem cells via modulation of sonic hedgehog pathway. Acta Biochim Biophys Sin (Shanghai) 50: 586-596, 2018.

54. Li SH, Fu J, Watkins DN, Srivastava RK and Shankar S: Sulforaphane regulates self-renewal of pancreatic cancer stem cells through the modulation of Sonic hedgehog-GLI pathway. Mol Cell Biochem 373: 217-227, 2013.

55. Zhou W, Hao M, Du X, Chen K, Wang G and Yang J: Advances in targeted therapy for osteosarcoma. Discov Med 17: 301-307, 2014.

56. Kun-Peng Z, Xiao-Long M and Chun-Lin Z: Overexpressed circPVT1, a potential new circular RNA biomarker, contributes to doxorubicin and cisplatin resistance of osteosarcoma cells by regulating ABCB1. Int J Biol Sci 14: 321-330, 2018.

57. Wang ZD, Wang RZ, Xia YZ, Kong LY and Yang L: Reversal of multidrug resistance by icaritin in doxorubicin-resistant human osteosarcoma cells. Chin J Nat Med 16: 20-28, 2018.

58. Qi XT, Li YL, Zhang YQ, Xu T, Lu B, Fang L, Gao JQ, Yu LS, Zhu DF, Yang B, et al: KLF4 functions as an oncogene in promoting cancer stem cell-like characteristics in osteosarcoma cells. Acta Pharmacol Sin 40: 546-555, 2019.

59. Vescovo T, Refolo G, Vitagliano G, Fimia GM and Piacentini M: Molecular mechanisms of hepatitis C virus-induced hepatocellular carcinoma. Clin Microbiol Infect 22: 853-861, 2016.

60. Wu Q, Chen JX, Chen Y, Cai LL, Wang XZ, Guo WH and Zheng JF: The chemokine receptor CCR10 promotes inflammation-driven hepatocarcinogenesis via PI3K/Akt pathway activation. Cell Death Dis 9: 232, 2018.

61. Yan HX, Wu HP, Zhang HL, Ashton C, Tong C, Wu H, Qian QJ, Wang HY and Ying QL: p53 promotes inflammation-associated hepatocarcinogenesis by inducing HMGB1 release. J Hepatol 59: 762-768, 2013.

62. Huan HB, Wen XD, Chen XJ, Wu L, Wu LL, Zhang L, Yang DP, Zhang X, Bie P, Qian C and Xia F: Sympathetic nervous system promotes hepatocarcinogenesis by modulating inflammation through activation of alpha1-adrenergic receptors of Kupffer cells. Brain Behav Immun 59: 118-134, 2017.

63. Li C, Deng M, Hu J, Li X, Chen L, Ju Y, Hao J and Meng S Chronic inflammation contributes to the development of hepatocellular carcinoma by decreasing miR-122 levels. Oncotarget 7: 17021-17034, 2016

64. Jung IH, Choi JH, Chung YY, Lim GL, Park YN and Park SW: Predominant activation of JAK/STAT3 pathway by interleukin-6 is implicated in hepatocarcinogenesis. Neoplasia 17: 586-597, 2015.

This work is licensed under a Creative Commons Attribution-NonCommercial-NoDerivatives 4.0 International (CC BY-NC-ND 4.0) License. 